\title{
Water-mediated interactions determine helix formation of peptides in open nanotubes
}

\author{
Dylan Suvlu, ${ }^{\dagger}$ D. Thirumalai, ${ }^{\ddagger}$ and Jayendran C. Rasaiah* ${ }^{*} \dagger$ \\ $\dagger$ University of Maine, Orono, ME 04469 \\ $\ddagger$ The University of Texas at Austin, Austin, TX 78712
}

E-mail: rasaiah@maine.edu 


\begin{abstract}
Water-mediated interactions (WMIs) play diverse roles in molecular biology. They are particularly relevant in geometrically confined spaces such as the interior of the chaperonin, at the interface between ligands and their binding partners, and in the ribosome tunnel. Inspired in part by the geometry of the ribosome tunnel, we consider confinement effects on the stability of peptides. We describe results from replica exchange molecular dynamics simulations of a system containing a 23-alanine or 23serine polypeptide confined to non-polar and polar nanotubes in the gas phase and when open to a water reservoir. We quantify the effect of water in determining the preferred conformational states of these polypeptides by calculating the difference in the solvation free energy for the helix and coil states in the open nanotube in the two phases. Our simulations reveal several possibilities. We find that nanoscopic confinement preferentially stabilizes the helical state of polypeptides with hydrophobic side chains, which is explained by the entropic stabilization mechanism proposed on the basis of polymer physics. Polypeptide chains with hydrophilic side chains can adopt helical structures within nanotubes, but helix formation is sensitive to the nature of the nanotube due to WMIs. We elaborate on the potential implications of our findings to the stability of peptides in the ribosome tunnel.
\end{abstract}

\title{
Introduction
}

Understanding proteins in crowded and confining environments is important for knowledge of their function in the cell. ${ }^{1-3}$ For example, experiments suggest that the architecture of the ribosome promotes transient structure acquisition in newly synthesized proteins. ${ }^{4 ?-12}$ Theoretical studies predicted that the ribosome tunnel stabilizes the $\alpha$-helix in proteins by destabilizing the coiled state through a reduction in entropy of the coiled state due to conformational restrictions from the confining walls. ${ }^{13-15}$ However, molecular dynamics simulations of a hydrophobic polypeptide confined to a periodically replicated carbon nanotube contain- 
ing water showed that the coiled state is preferred in the nanotube, ${ }^{16}$ which appears to naively contradict the expectations based on polymer physics. ${ }^{13}$ The cause of this surprising behavior was attributed to the presence of water which was confined within the nanotube. ${ }^{17}$ Removal of periodic replication of the nanotube and exposure to a water reservoir completely changes the picture, and the conditions for helix formation now occurs within a range of hydrophobicity and tube diameters similar to the diameters of the ribosome tunnel. ${ }^{18}$ Additional studies indicate a central role for water in contributing to conformational preferences of biomolecules in crowded and confining environments, ${ }^{19-23}$ and water has been receiving increasing attention for its role in biology. For example, the hydrophobic effect, studied in a series of pioneering papers initiated by Pratt, ${ }^{24,25}$ is thought to play a major role in protein folding. ${ }^{26-29}$ Further, water-mediated interactions (WMIs) have been demonstrated to play a role in protein aggregation and amyloid formation and amyloid polymorphism. ${ }^{30,31}$ It has even been speculated that water may be the universal solvent for life. ${ }^{32}$ However, WMIs are not completely understood, and surprises ${ }^{33,34}$ and new perspectives continue to emerge $^{35}$ largely because the variability in water-mediated interactions are dictated by the context. Therefore, WMIs continue to be an abiding topic of interest and characterizing their influence remains to be a fruitful enterprise. ${ }^{36-39}$

Conformational preferences of biomolecules in nanoscopic confinement are difficult to predict due to the presence of many competing interactions. For example, one must consider not only intramolecular interactions within the protein, but also intermolecular interactions with the confining surface and how both of these interactions are mediated by water within and outside a nanotube open to a reservoir. The latter water-mediated component of these interactions is inherently a many-body effect, thus making it difficult to describe theoretically. Further, water can behave in surprising ways in confined spaces ${ }^{40-43}$ leading to additional complexity. Here, we employ a model system containing a homopolymer with either a hydrophobic or hydrophilic amino acid confined to polar or non-polar nanotubes in the gas phase and in the solution phase when the nanotubes are open to a water reservoir. 
At equilibrium, the chemical potential of water within and outside the open nanotube in solution is identical. The model benefits from being simple enough to extract meaningful trends, but complex enough to potentially be relevant to biological systems. Using molecular dynamics simulations, we demonstrate that water-mediated interactions (WMIs) contribute to conformational preferences of polypeptides in nanotubes. We quantify WMIs in this context by calculating the difference in the solvation thermodynamics between the helix and coil states while confined to non-polar and polar nanotubes in the gas and solution phases. Our simulation results suggest that hydrophobic sequences preferentially form $\alpha$ helices inside open nanotubes. The results presented here should be of interest to researchers studying water-mediated interactions and confinement effects on biomolecules.

\section{Methods}

Our liquid water simulations followed a similar procedure to our previous work. ${ }^{18}$ We performed replica exchange molecular dynamics simulations ${ }^{44}$ using GROMACS $5.1 .2^{45}$ with the CHARMM36 force field. ${ }^{46,47}$ The nanotubes were $100 \AA$ in length with diameters $(D=$ $0.783 \AA \sqrt{3} n) 13.6,14.9,16.3,17.6$, and $18.9 \AA$. The 13.6 and $14.9 \AA$ NT systems each contained 74 replicas, while the $16.3,17.6$, and $18.9 \AA$ NT systems each contained 84 replicas. The replicas spanned the temperature range $280 \mathrm{~K}$ to $500 \mathrm{~K}$ with the temperature spacing between replicas determined by a procedure described elsewhere ${ }^{48}$ such that the exchange probability between replicas was at least $0.23( \pm 0.02)$. Exchange attempts between replicas were attempted every 1 ps. Errors were estimated with block averaging. ${ }^{49}$ The gas phase simulations followed a procedure used previously to study protein dynamics in the gas phase. ${ }^{50,51}$ The number of replicas for each NT system in the gas phase was 30 , spanning the temperature range $280 \mathrm{~K}$ to $600 \mathrm{~K}$ with temperature spacing such that the exchange probability between replicas was approximately $0.30( \pm 0.01)$. Additional details of the simulation methods can be found in the Supporting Information. 


\section{Results and Discussion}

To compute the difference in the solvation free energies between the helix (h) and coil (c) states, we constructed a thermodynamic cycle (Fig. 1). The solvation free energy of the helix is $\Delta G_{h}^{s}=\Delta H_{h}^{s}-T \Delta S_{h}^{s}$ where $\Delta H_{h}^{s}=E_{h}^{u v}+\Delta H_{h}^{v v}$ and $\Delta S_{h}^{s}=S_{h}^{u v}+\Delta S_{h}^{v v} \cdot{ }^{52-55}$ The solute (polypeptide + nanotube) is denoted by $u$ and the solvent (water) is denoted by $v$. The quantity $E_{h}^{u v}$ is the average interaction energy between the solute and solvent, and $\Delta H_{h}^{v v}$ is the water reorganization energy upon solvation of the helical state. ${ }^{52-55}$ Further, the water reorganization energy is exactly compensated by the water reorganization entropy, $\Delta H_{h}^{v v}=T \Delta S_{h}^{v v} \cdot{ }^{52-55}$ The quantity $S_{h}^{u v}$ is the corresponding entropy associated with solutesolvent interaction energy fluctuations. ${ }^{52-55} \mathrm{~A}$ similar expression holds for the coil. The difference in solvation free energies is,

$$
\begin{aligned}
\Delta \Delta G_{h c} & =\Delta G_{h}^{s}-\Delta G_{c}^{s} \\
& =\Delta \Delta H-T \Delta \Delta S \\
& =\Delta E_{h c}^{u v}+\Delta \Delta H_{h c}^{v v}-T\left(\Delta S_{h c}^{u v}+\Delta \Delta S_{h c}^{v v}\right) .
\end{aligned}
$$

Because the Gibbs free energy is a state function, the difference in solvation free energies can be computed through the difference in the free energies of the helix and coil states in the liquid $\left(\Delta G_{h c}^{l}\right)$ and gas phases $\left(\Delta G_{h c}^{g}\right), \Delta \Delta G_{h c}=\Delta G_{h}^{s}-\Delta G_{c}^{s}=\Delta G_{h c}^{l}-\Delta G_{h c}^{g}$. Both $\Delta G_{h c}^{l}$ and $\Delta G_{h c}^{g}$ can be obtained from replica exchange molecular dynamics simulations between identical systems over a range of temperatures, from which we obtain an estimate of $\Delta \Delta G_{h c}$ and, therefore, insight into the water-mediated contributions to helix formation inside nanotubes open to a water reservoir.

We computed $\Delta \Delta G_{h c}$ for four systems. (1) A polypeptide $\left(\mathrm{A}_{23}\right)$ with a non-polar side chain $\left(-\mathrm{CH}_{3}\right)$ confined to a non-polar carbon nanotube (NPNT). (2) A polypeptide $\left(\mathrm{S}_{23}\right)$ with a polar side chain $\left(-\mathrm{CH}_{2} \mathrm{OH}\right)$ confined to a non-polar carbon nanotube. (3) $\mathrm{A}_{23}$ confined to a polar boron nitride nanotube $(\mathrm{PNT})$, and (4) $\mathrm{S}_{23}$ confined to a polar boron nitride 


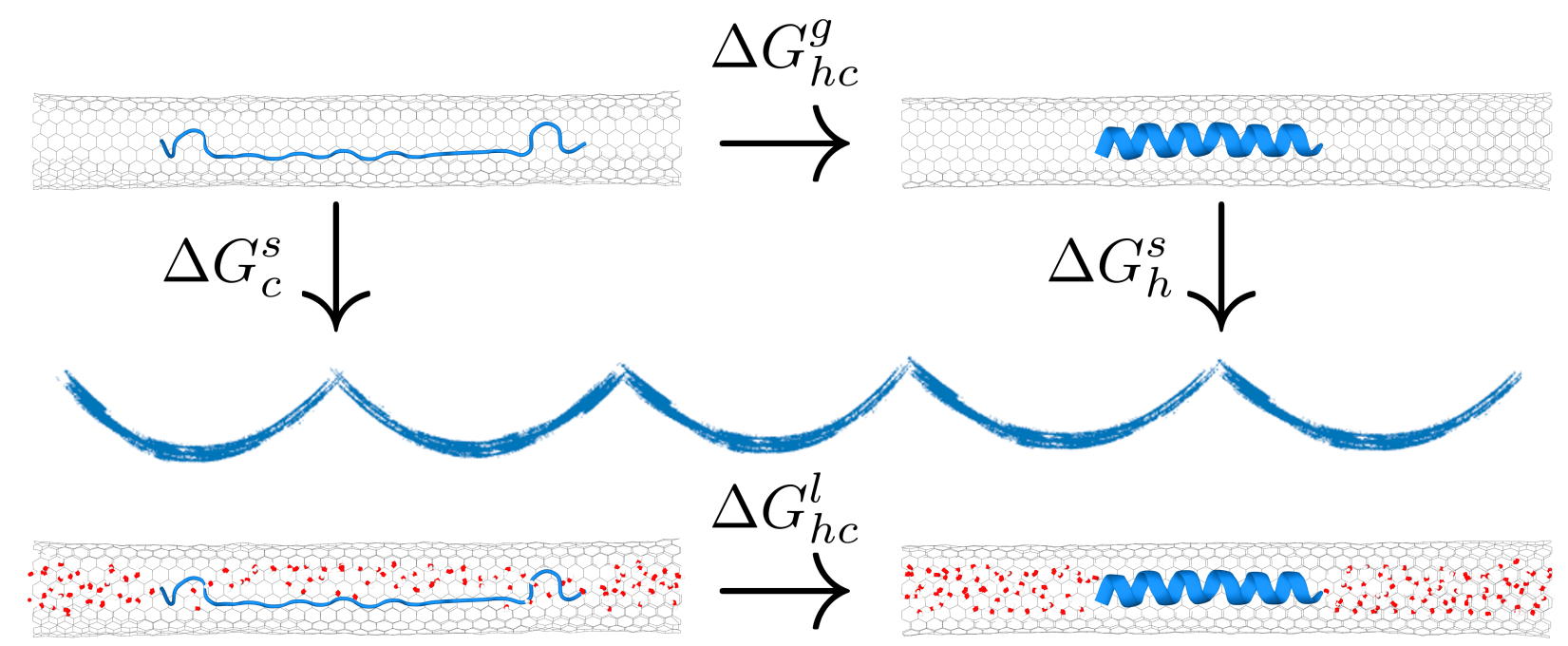

Figure 1: Thermodynamic cycle used in calculating $\Delta \Delta G_{h c}$, the difference in solvation free energies between the helix and coil states in the gas and liquid phases.

nanotube.

The free energy of helix formation in the liquid and gas phases was computed as $\Delta G_{h c}^{x}=$ $-R T \ln K(T)$ where the temperature dependent equilibrium ratio $K(T)=f_{h}(T) /\left(1-f_{h}(T)\right)$. The fraction of the polypeptide in the helix state $f_{h}$ is obtained through a Lifson-Roig formalism for helix-random coil transition of polypeptides as was done in our previous publication ${ }^{18}$

$$
f_{h}(T)=\frac{1}{n-2} \sum_{i=2}^{n-1} \frac{\partial \ln Z(T)}{\partial \ln w_{i}(T)}
$$

where $Z$ is the partition function of the polypeptide, $n$ is the number of amino acids, and $w_{i}(T)$ is a weight factor for each amino acid. ${ }^{56}$ Additional details about how the thermodynamics at $300 \mathrm{~K}$ were determined are provided in the Supporting Information.

Figure 2 displays the fraction helix of $\mathrm{A}_{23}$ and $\mathrm{S}_{23}$ confined to the NPNT and PNT with diameters $13.6 \AA$ and $14.9 \AA$, in the liquid and gas phases as a function of temperature. Inside both the non-polar nanotube (NPNT) and polar nanotube (PNT), $\mathrm{A}_{23}$ has greater helix content in the presence of water compared to the gas phase. Conversely, at $300 \mathrm{~K} \mathrm{~S}_{23}$ forms a helix inside the NPNT in the gas phase but not in the presence of water. However, the helix content of $\mathrm{S}_{23}$ is reestablished in the PNT when in the presence of water. We 
elaborate on these observations by discussing the difference in free energies of helix and coil states in the gas and liquid phases $\Delta \Delta G_{h c}$ for the four systems.
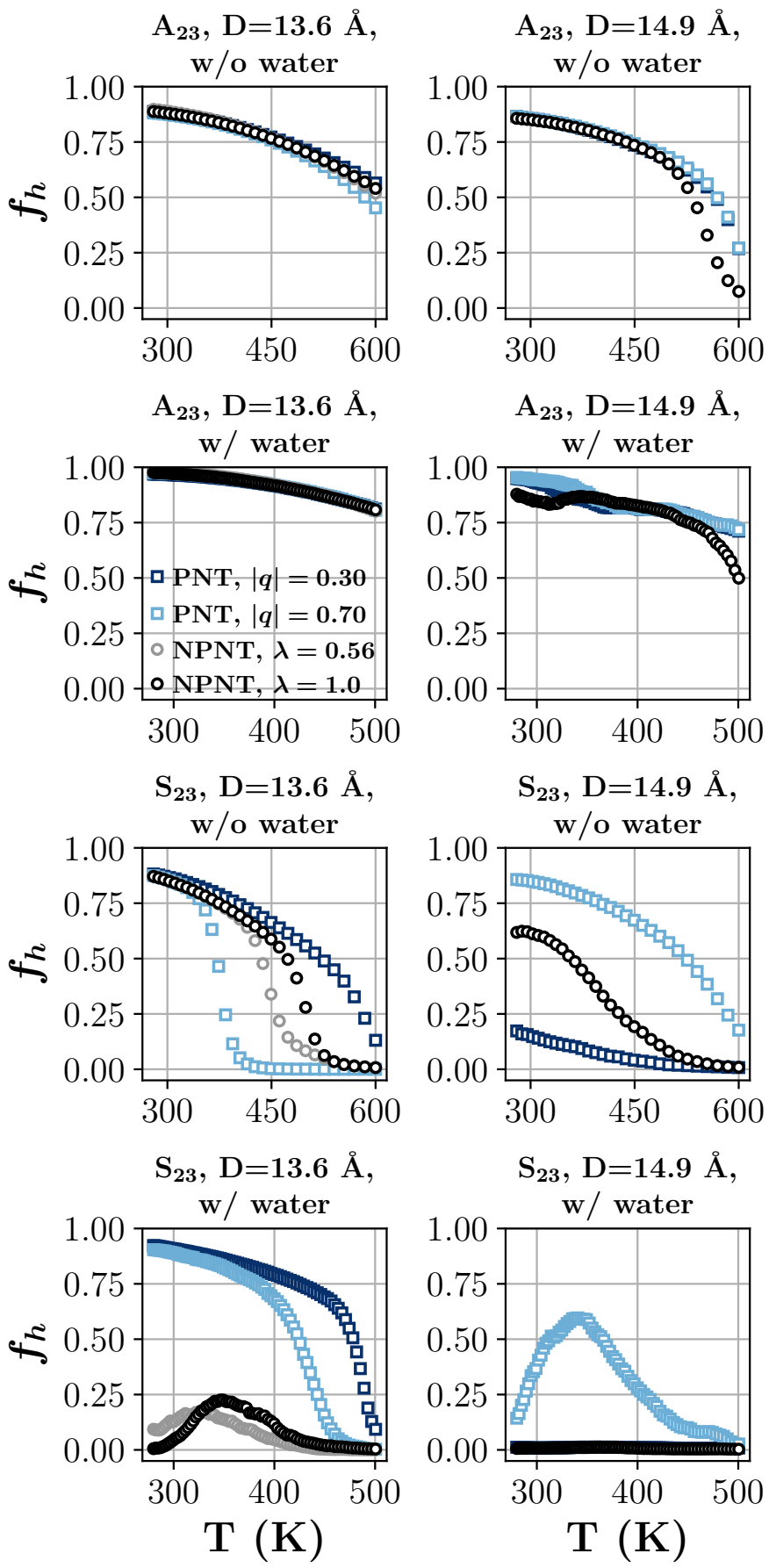

Figure 2: Fraction helix $f_{h}$ of polyalanine $\left(\mathrm{A}_{23}\right)$ and polyserine $\left(\mathrm{S}_{23}\right)$ as a function of temperature while confined inside the non-polar carbon nanotube (NPNT) and polar boron nitride nanotube (PNT) for diameters $D=13.6 \AA$ (left column) and $D=14.9 \AA$ (right column) both with and without water. 


\section{$\mathrm{NPNT} / \mathbf{A}_{23}$}

We calculated the free energy of helix formation in $\mathrm{A}_{23}$ inside a carbon nanotube in the gas phase and in liquid water (Fig. 3). Compared to the gas phase, the helix state of $\mathrm{A}_{23}$ in the presence of water has a lower free energy with $\Delta \Delta G_{h c} \approx-4 \mathrm{~kJ} / \mathrm{mol}$ (Table 1 ). In other words, it is more favorable to solvate the $A_{23}$ helix/nanotube system than the $A_{23}$ coil/nanotube system. Table 1 suggests that this is an energetic rather than an entropic effect. Specifically, $\Delta \Delta H<0$ while $\Delta \Delta S<0$. However, upon closer inspection we will find that this must be driven by the entropy. In the coil state, $\mathrm{A}_{23}$ has many more hydrogen bonds with water than in the helix state. Therefore, the water-polypeptide interaction energy is expected to be more negative for the coil than the helix, $\Delta E^{u v}=E_{h}^{u v}-E_{c}^{u v}>$ 0. Consequently, the difference in water reorganization energy between the helix and the coil states must be negative, $\Delta \Delta H_{h c}^{v v}<0$. See the Supporting Information for further evidence of this. Accordingly, one might expect water reorganization energy to favor the helix state inside the nanotube, and provide greater helix stability for $\mathrm{A}_{23}$ in the liquid phase compared to the gas phase. However, the water reorganization energy and entropy exactly compensate, $\Delta H^{v v}=T \Delta S^{v v}$, as discussed above. Therefore, $\Delta \Delta G_{h c}=\Delta E_{h c}^{u v}-T \Delta S_{h c}^{u v}$. For $\Delta \Delta G_{h c}<0$, as is the case for $\mathrm{A}_{23}$ confined to the NPNT, the entropy associated with the fluctuations in the solute-solvent interaction energy must be positive, $\Delta S_{h c}^{u v}>0$. Thus, the greater helix stability conferred to the peptide in the liquid phase compared to the gas phase originates from the positive entropy change associated with the fluctuations in the water-solute interaction energy.

We also scaled the dispersion energy of the carbon nanotube with a parameter $\lambda$ as in our previous study. ${ }^{18}$ The free energy of helix formation inside the NPNT in the gas phase is relatively insensitive to reductions in the dispersion energy. In the presence of water, however, the free energy of helix formation decreases by approximately $1 \mathrm{~kJ} / \mathrm{mol}$ as $\lambda$ decreases from 1.0 to 0.56 . The increase in helix stability as the dispersion energy of the nanotube decreases suggests a dewetting phenomena contributes to helix formation in this case. The surface of 

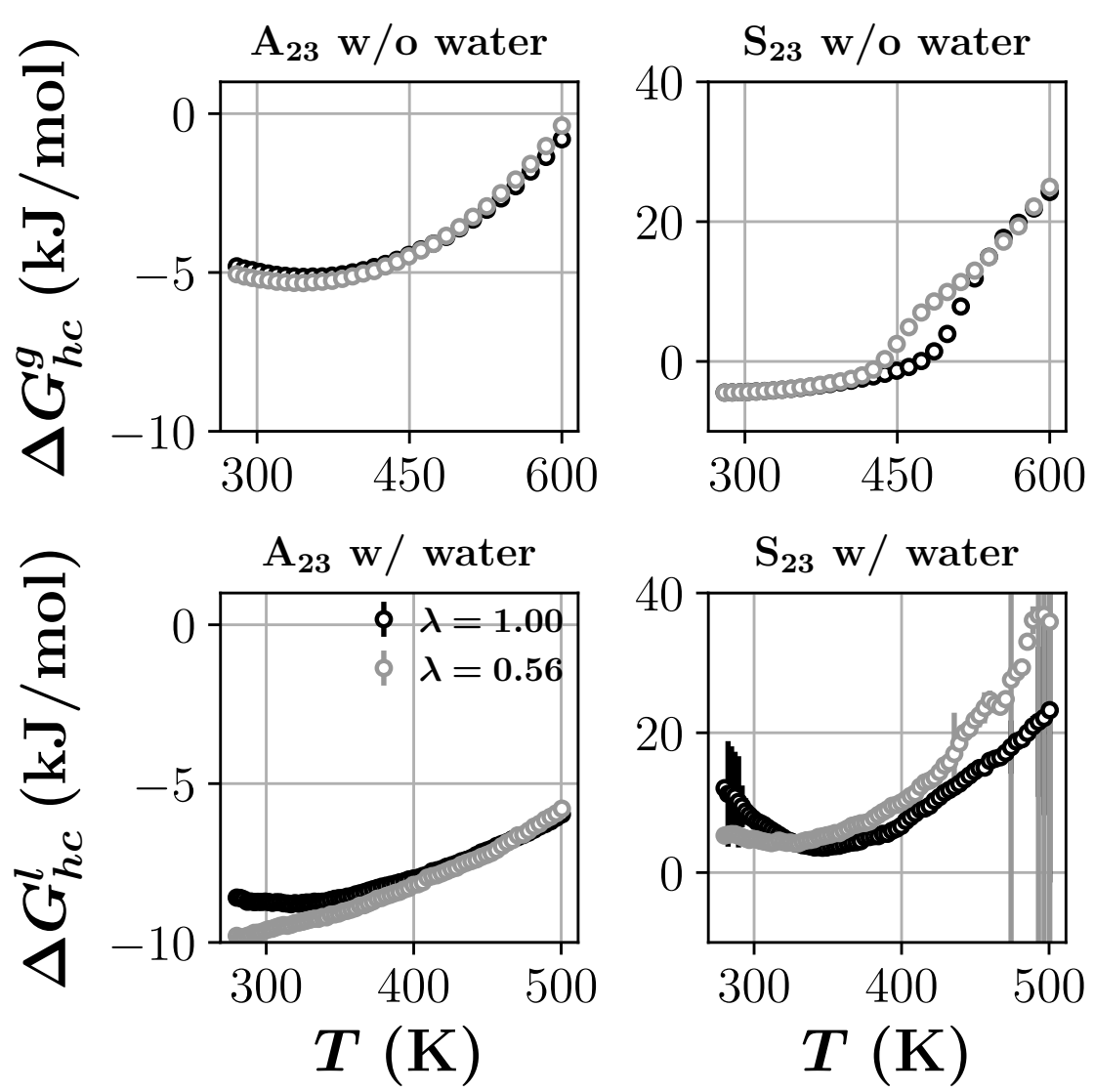

Figure 3: Free energy of helix formation in polyalanine $\left(\mathrm{A}_{23}\right)$ and polyserine $\left(\mathrm{S}_{23}\right)$ confined inside $13.6 \AA$ non-polar carbon nanotubes both with and without water. The parameter $\lambda$ scales the Lennard-Jones potential of the carbon nanotube.

the $\mathrm{A}_{23}$ helix is hydrophobic (Fig. 4) as is the inside surface of the NPNT. During helix formation, these two surfaces associate while simultaneously expelling water from the region between them into the reservoir. This dewetting phenomena becomes more likely as the dispersion energy of the nanotube decreases since there are fewer water molecules in the tube and relatively large water density fluctuations become more likely. ${ }^{27,57,58}$

\section{$\mathrm{NPNT} / \mathbf{S}_{23}$}

In contrast to $\mathrm{A}_{23}$, which has a hydrophobic side chain, $\mathrm{S}_{23}$ has a hydrophilic side chain (Fig. 4). Therefore, the water-mediated interactions between $\mathrm{S}_{23}$ and the nanotube are expected to be different from $\mathrm{A}_{23}$. Indeed, we observe helix formation in $\mathrm{S}_{23}$ in the NPNT in 
Table 1: Differences in free energy, enthalpy, and entropy for the helix and coil states at $300 \mathrm{~K}$ for $\mathbf{A}_{23}$ and $\mathbf{S}_{23}$ confined to the non-polar carbon nanotube of diameter $D=13.6 \AA$.

\begin{tabular}{rrrr}
\hline & $\Delta \Delta G_{h c}(\mathrm{~kJ} / \mathrm{mol})$ & $\Delta \Delta H(\mathrm{~kJ} / \mathrm{mol})$ & $\Delta \Delta S(\mathrm{~J} / \mathrm{mol})$ \\
\hline \multicolumn{4}{c}{$\mathrm{A}_{23}$} \\
$\lambda=1.00$ & $-3.79 \pm 0.25$ & $-4.42 \pm 0.18$ & $-2.1 \pm 0.6$ \\
$\lambda=0.56$ & $-4.42 \pm 0.39$ & $-9.29 \pm 0.27$ & $-16.2 \pm 0.9$ \\
\multicolumn{4}{c}{$\mathrm{S}_{23}$} \\
$\lambda=1.00$ & $11.1 \pm 2.3$ & $48.8 \pm 1.7$ & $125.6 \pm 5.2$ \\
$\lambda=0.56$ & $9.2 \pm 1.5$ & $25.8 \pm 1.1$ & $55.3 \pm 3.4$ \\
\hline
\end{tabular}

the gas phase at $300 \mathrm{~K}$ which is expected from polymer physics. However, we do not observe helix formation in $\mathrm{S}_{23}$ the presence of water inside the NPNT. This is further demonstrated by the negative change in free energy for helix formation at $300 \mathrm{~K}$ in the gas phase, but positive free energy change for helix formation in the presence of water (Fig. 3). The result is a positive difference in free energies between the helix and coil states for $\mathrm{S}_{23}$ (Table 1). In other words, solvation of the $\mathrm{S}_{23}$ coil/nanotube system is more favorable than the $\mathrm{S}_{23}$ helix/nanotube system when the nanotube has non-polar walls. The $-\mathrm{OH}$ functional group of $\mathrm{S}_{23}$ forms approximately 42 hydrogen bonds with water molecules inside the $D=13.6 \AA$ nanotube (Fig. 5). To form a helix, $\mathrm{S}_{23}$ would have to break many of these hydrogen bonds because at $D=13.6 \AA$ there is not enough room inside the nanotube to maintain a hydration layer around the helix (Fig. 4). Therefore, $\Delta E_{h c}^{u v}>>0$ and $\Delta \Delta H>>0$. Further evidence of this is displayed in the Supporting Information. Consequently, helix formation is not favored in $\mathrm{S}_{23}$ inside the NPNT. The release of the hydrogen bonded water from the serine hydroxyl group during helix formation would result in an increase in the entropy of the bound water due to the increase in translational freedom. This is reflected in the positive $\Delta \Delta S$ in Table 1, but its magnitude is not large enough to compensate for the energy required to desolvate the peptide side chain. In this case, it could be expected that the helix content in $\mathrm{S}_{23}$ might increase with temperature as water's tendency to form hydrogen bonds decreases with increasing temperature. This is observed in the temperature dependence of the helix 


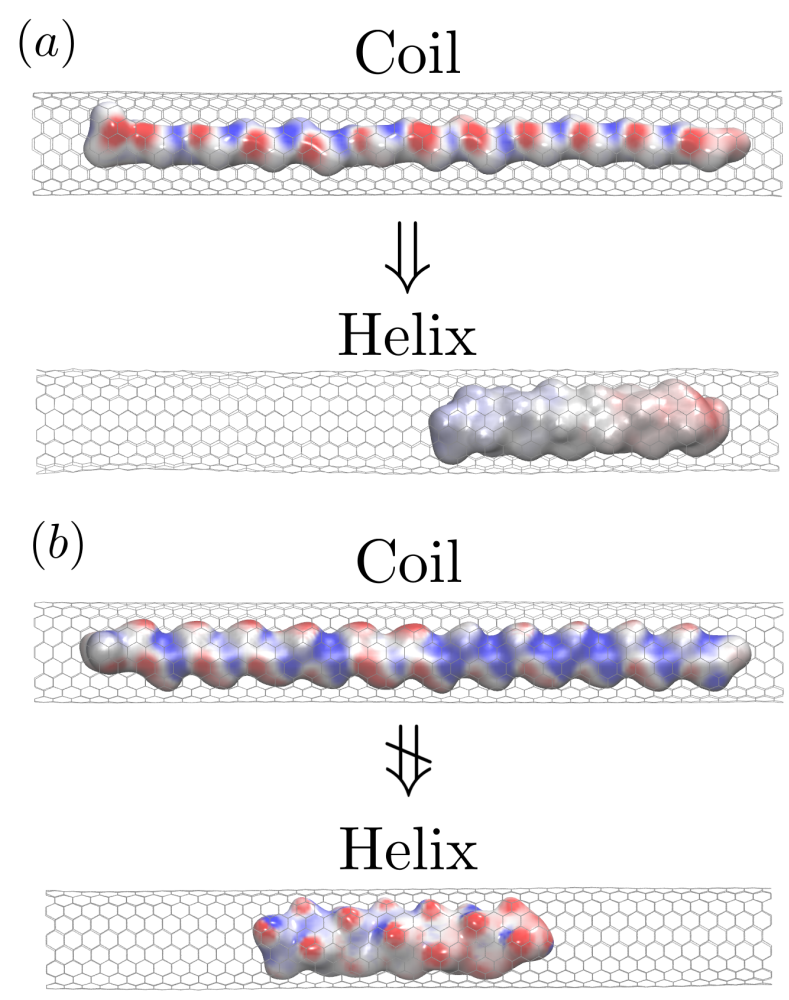

Figure 4: Electrostatic potential from the Poisson-Boltzmann equation for the coil and helix states for (a) $\mathrm{A}_{23}$ and (b) $\mathrm{S}_{23}$ inside the NPNT. Blue indicates positively polarized ( $\left.-\mathrm{NH}\right)$, red indicates negatively polarized $(-\mathrm{CO}$ and $-\mathrm{OH})$, and gray indicates neutral or non-polar. Note the non-polar nature of the surface of the $\mathrm{A}_{23}$ helix, and the polar nature of the surface of the $\mathrm{S}_{23}$ helix.

content in $\mathrm{S}_{23}$ in the NPNT, with a maximum in $f_{h}$ observed at approximately $350 \mathrm{~K}$ before approaching zero for $T>350 \mathrm{~K}$ (Fig. 2).

\section{$\mathrm{PNT} / \mathbf{A}_{23}$}

The temperature dependence of $f_{h}$ is very similar in the PNT/A $\mathrm{A}_{23}$ system compared to the NPNT/ $\mathrm{A}_{23}$ system, with minor differences observed at a larger nanotube diameter of $D=14.9 \AA$ (Fig. 2). The difference in the solvation free energies between the helix and coil states is approximately the same in both nanotubes with $\Delta \Delta G_{h c} \approx-4 \mathrm{~kJ} / \mathrm{mol}$ (Table 2). However, there are disparities in the enthalpy and entropy differences in the two systems. For example, $\Delta \Delta H$ is less negative in the PNT than the NPNT, but this is compensated by a positive $\Delta \Delta S$ in the PNT suggesting the disparities in the thermodynamics arise from 

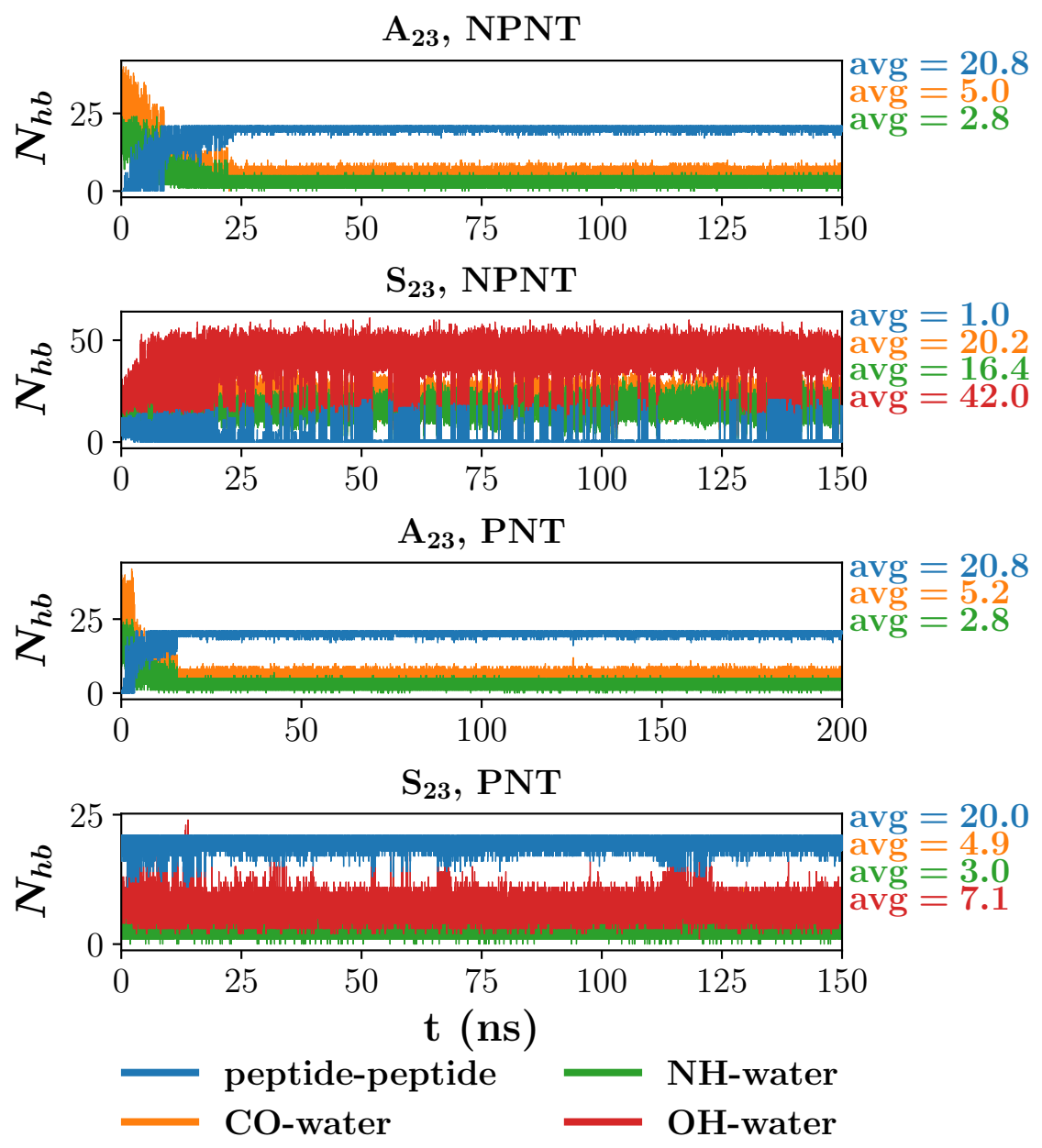

Figure 5: Number of peptide-peptide and peptide-water hydrogen bonds $\left(N_{h b}\right)$ as a function of time at $300 \mathrm{~K}$ for $\mathrm{A}_{23}$ and $\mathrm{S}_{23}$ confined to the $\operatorname{NPNT}(\lambda=1.00)$ and $\operatorname{PNT}(|q|=0.30)$. The displayed averages are calculated from the last $50 \mathrm{~ns}$ of the simulations.

compensating water reorganization enthalpy and entropy. See the Supporting Information for further discussion. Thus, $\mathrm{A}_{23}$ appears agnostic to the nature of the nanotube surface, at least for a tube with relatively small partial charges without hydrogen-bonding groups. Surprisingly, the difference in free energies between helix and coil states $\Delta \Delta G_{h c}$ becomes more negative as the partial charge on the boron and nitrogen atoms increase inside the nanotube. This is likely because the water removed from the nanotube surface during helix formation in the $|q|=0.70$ system has a larger entropy change $\Delta S_{h c}^{u v}$ than the $|q|=0.30$ system. 

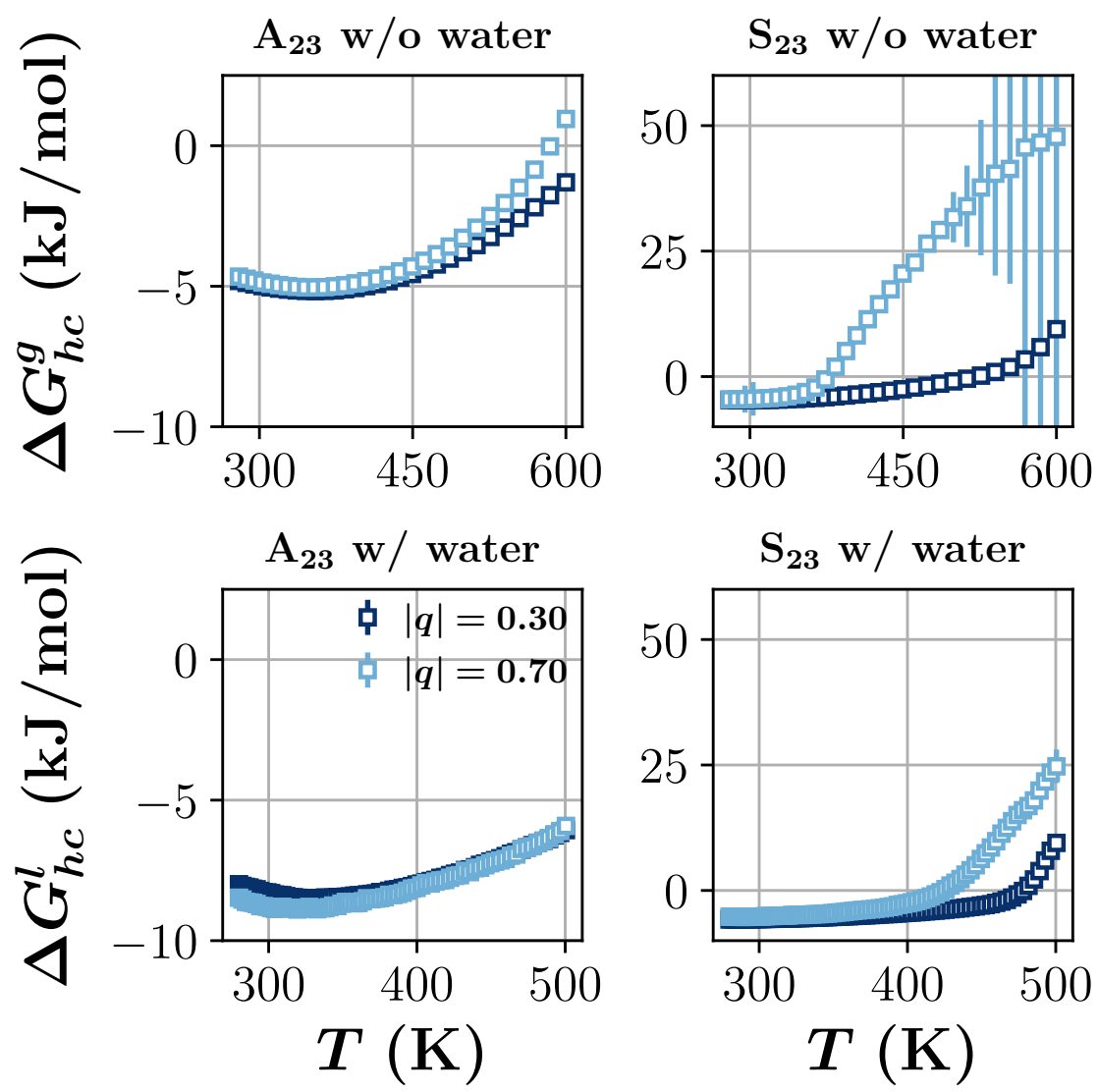

Figure 6: Free energy of helix formation in polyalanine and polyserine confined to a $13.6 \AA$ boron nitride nanotube both with and without water. $|q|$ is the absolute value of the partial charge placed on the boron and nitrogen atoms in the boron nitride nanotube.

\section{$\mathrm{PNT} / \mathrm{S}_{23}$}

Upon changing the nanotube walls from non-polar to polar, $\mathrm{S}_{23}$ forms a helix when confined to the $D=13.6 \AA$ nanotube in the presence of water at $300 \mathrm{~K}$. Further, the helix is more stable in the presence of water than in the gas phase with $\Delta \Delta G_{h c} \approx-1 \mathrm{~kJ} / \mathrm{mol}$. The thermodynamics in the PNT (Table 2) demonstrate a dramatic reversal from $\mathrm{S}_{23}$ in the NPNT. Instead of a large positive enthalpy change as in the NPNT, the partial charge on the PNT has made it so $\Delta \Delta H<0$. The electrostatic interaction between the peptide and nanotube mitigates the desolvation energy of the peptide upon helix formation (see the SI for further discussion), thereby stabilizing the helix state in the PNT. When $|q|=0.70$ the uncertainty resulting from the fit to $\ln K(T)$ in the gas phase was too large to provide 
accurate estimates of the thermodynamics. With a slight increase in the nanotube diameter from $D=13.6 \AA$ to $D=14.9 \AA$, the fraction of $\mathrm{S}_{23}$ forming a helix is nearly zero (Fig. 2) in the presence of water except in the nanotube with $|q|=0.70$ partial charges on boron and nitrogen.

Table 2: Differences in solvation free energy, enthalpy, and entropy for the helix and coil states at $300 \mathrm{~K}$ for $\mathrm{A}_{23}$ and $\mathrm{S}_{23}$ confined to the polar boron nitride nanotube.

\begin{tabular}{rrrr}
\hline & $\Delta \Delta G_{h c}(\mathrm{~kJ} / \mathrm{mol})$ & $\Delta \Delta H(\mathrm{~kJ} / \mathrm{mol})$ & $\Delta \Delta S(\mathrm{~J} / \mathrm{mol})$ \\
\hline \multicolumn{4}{c}{$\mathrm{A}_{23}$} \\
$|q|=0.30$ & $-3.33 \pm 0.33$ & $-2.03 \pm 0.23$ & $4.31 \pm 0.34$ \\
$|q|=0.70$ & $-3.95 \pm 0.37$ & $-3.78 \pm 0.26$ & $0.60 \pm 0.87$ \\
\multicolumn{4}{c}{$\mathrm{S}_{23}$} \\
$|q|=0.30$ & $-1.09 \pm 0.62$ & $-4.85 \pm 0.44$ & $-12.6 \pm 1.4$ \\
$|q|=0.70$ & $-0.9 \pm 3.5$ & $3.2 \pm 2.5$ & $13.3 \pm 8.2$ \\
\hline
\end{tabular}

\section{NPNT/VSV-G}

We also conducted gas phase and liquid water replica exchange MD simulations of wild type and three mutant amino acid sequences from the membrane protein VSV-G. The 20 amino acid wild type sequence was SSIASFFFIIGLIIGLFLVL. In the first mutant (AAIAAFFFIIGLIIGLFLVL) we substituted alanine for serine to observe what effect, if any, the substitution would have on the helix content of the protein sequence. In the second mutant (SSIASFFFIIALIIALFLVL) we substituted alanine for glycine since glycine is a known helix breaker in proteins. The third mutant contained both substitutions (AAIAAFFFIIALIIALFLVL). Figure 7 displays $f_{h}$ for each sequence as a function of nanotube diameter and protein sequence both with and without water while confined to the NPNT. In each case, the middle of the sequence forms a helix inside the nanotube with slight helicity towards the C-terminus of the sequence. This observation is consistent with results from molecular dynamics simulations of the same sequence confined to the ribosome tunnel. ${ }^{11}$ The N-terminus of the sequence contains three 

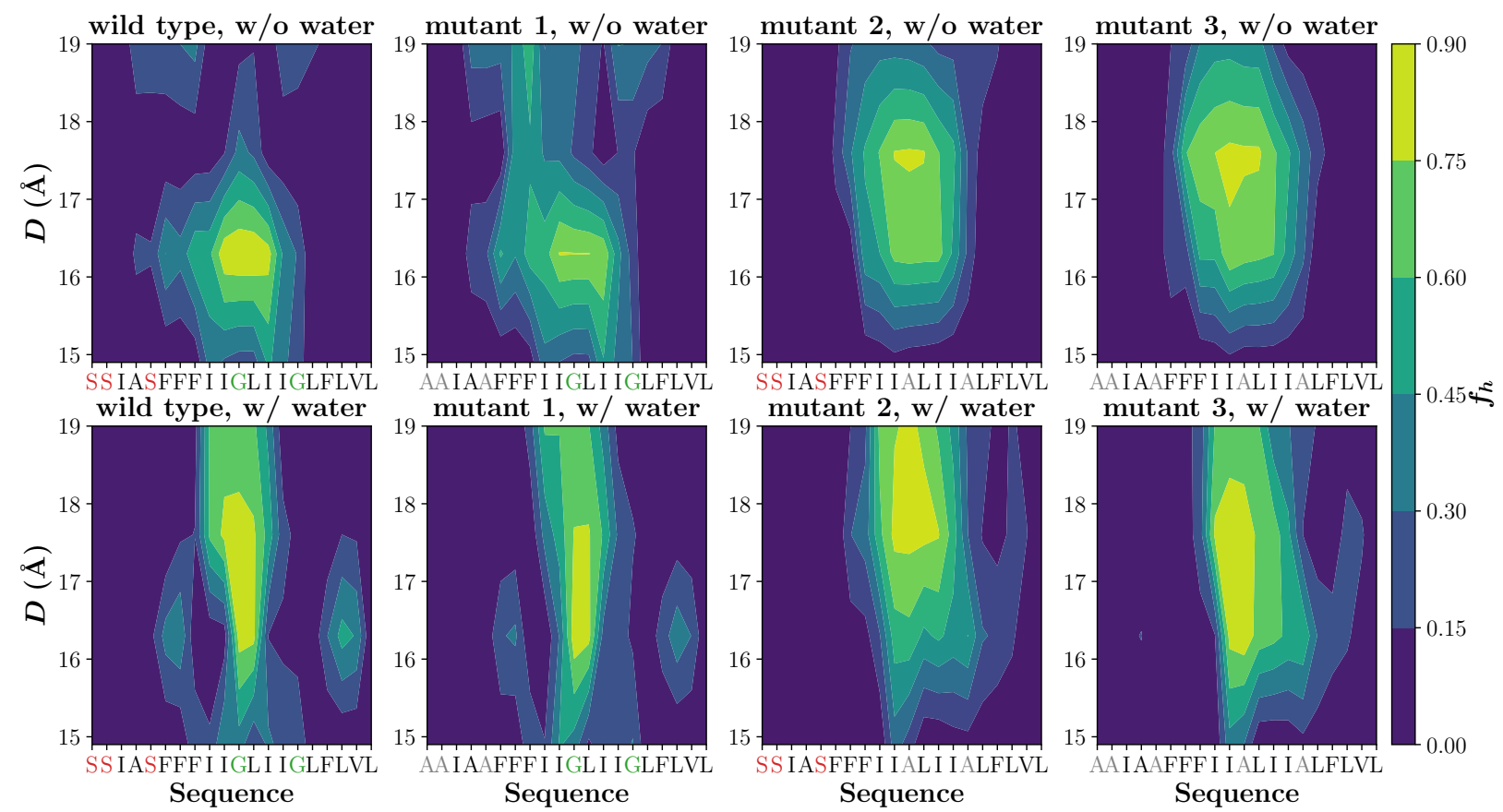

Figure 7: Displayed is the fractional helix content $f_{h}$ of wild-type and mutant sequences extracted from the protein VSV-G as a function of nanotube diameter and protein sequence. The top row is from simulations in the gas phase, while the simulations in the second row contained liquid water.

serines that display little helicity inside the nanotube, and does not increase upon substitution with alanine. Confinement has the greatest effect on helix stabilization in alanine and serine at $D=13.6 \AA$ while the amino acids with bulky side chains, such as isoleucine (I) and leucine (L), are too large to form a helix at that diameter. Therefore, we do not observe helix formation in alanine or serine for $D \geq 14.9 \AA$ but we observe helix formation in the bulky amino acids in the middle of the protein sequence. These data highlight the extreme sensitivity of confinement induced helix stabilization on the diameter of the confining surface.

\section{Biological Implications}

The results presented thus far demonstrate a complex interplay of many competing interactions contributing to the preferred states of proteins in nanotubes. Confinement to the nanotube in the gas phase induces helix formation when the diameter of the confining wall is 
slightly larger than the diameter of the helix (Fig. 2). This behavior is expected from prior studies utilizing elegant concepts from polymer physics. ${ }^{13}$ However, even in the gas phase the extent of helix stabilization depends on the nature of the amino acid sequence and on the type of interactions with the nanotube walls. For example, polyalanine forms a helix inside both the non-polar and polar nanotubes, but polyserine displays differing behavior depending on the interactions with the nanotube, especially as the diameter of the nanotube increases (Fig. 2). In liquid water, water-mediated interactions between the protein and the nanotube further complicate the picture. For example, when the nanotube wall is nonpolar, polyserine prefers the coil state. However, upon confinement to a nanotube with polar walls, the helix state is stabilized for diameters just slightly larger than the helix. These data suggest that protein sequences containing stretches of hydrophobic amino acids might preferentially form $\alpha$-helices inside the ribosome tunnel. Indeed, experiments investigating helix formation inside the ribosome tunnel demonstrated that hydrophobic transmembrane protein sequences form compacted structures inside the tunnel, suggesting the formation of an $\alpha$-helix. ${ }^{11}$

We performed simulations of a 20 amino acid sequence from wild-type and mutant forms of the VSV-G protein confined to a non-polar nanotube with varying diameters. In the wildtype and mutant sequences we observed helix formation in the middle of the sequence with some helicity at the C-terminus. The portion of the protein sequence containing serine did not form a helix inside the nanotube and this was expected when considering our results with $\mathrm{S}_{23}$. When alanine was substituted for serine in the sequence, the helicity did not increase which was contrary to our expectations arising from the $\mathrm{A}_{23}$ results. These observations can be rationalized when considering the strong diameter dependence of confinement induced helix stabilization. The smaller alanine does not experience confinement induced helix stabilization at the same diameters as the larger hydrophobic amino acids such as leucine (L), isoleucine (I), and phenylalanine (F). Consequently, the helicity does not increase upon substitution of serine with alanine for $D \geq 14.9 \AA$. However, each VSV-G protein sequence formed a helix 
within the center of the sequence and this is consistent with prior studies. ${ }^{11}$

\section{Concluding Remarks}

In this paper, we present the results of replica exchange molecular dynamics to investigate the thermodynamics of helix-coil transitions of the polypeptides 23-alanine and 23-serine in open nanotubes. The transitions occur at diameters similar to the width of the ribosome tunnel, and are accompanied by the expulsion of water from the tubes when they are open to a water reservoir. We elucidate the effect of water by comparison with the corresponding results for systems in the gas phase. We also compare and contrast gas and liquid water phase replica exchange MD simulations of the wild type and mutant amino acid sequences from the membrane protein VSV-G.

Taken together, our results suggest that water-mediated interactions contribute to helix formation inside nanotubes. Several possibilities emerge for the water mediated interactions depending on the sequence of the peptides, which implies that it is difficult to construct a generic theory. However, we showed that helix formation is dependent on the difference in water interaction energy $\Delta E_{h c}^{u v}$ and entropy $\Delta S_{h c}^{u v}$ in the helix and coil states. Peptides containing stretches of hydrophobic amino acids preferentially form $\alpha$-helices inside nanotubes, which is consistent with theories based on polymer physics. Our simulations show that protein sequences containing polar amino acids could also form $\alpha$-helices but the extent of helix formation is sensitively dependent on the diameter of the confining nanotube and the nature of the nanotube surface. The different possibilities illustrated here also are consistent with experiments that show that structure formation in the ribosome tunnel depends on sequence. Our work shows that these variations are caused by water-mediated interactions, which implies that predicting the stability of proteins under confinement (in the cavity of GroEL or peptides in the ribosome tunnel) will require accounting for the effects of water. In this context, coarse-grained models incorporating water-mediated interactions ${ }^{59,60}$ may 
prove beneficial.

\section{Acknowledgement}

We thank Stephen Cousins of the University of Maine Advanced Computing Group for significant allotments of computing time and his technical assistance. This work was supported by the National Science Foundation (CHE 19-00093) and the Welch Foundation through grant F-0019 administered through the Collie-Welch chair.

\section{Supporting Information Available}

Additional details about the simulation methods and analysis, thermodynamics of helix formation for each system described in the main text, and interaction energies as a function of time and their relation to the solvation thermodynamics.

\section{References}

(1) Zhou, H.-X.; Rivas, G.; Minton, A. P. Macromolecular Crowding and Confinement: Biochemical, Biophysical, and Potential Physiological Consequences *. Annu. Rev. Biophys. 2008, 37, 375-397.

(2) Betancourt, M. R.; Thirumalai, D. Exploring the kinetic requirements for enhancement of protein folding rates in the GroEL cavity. J. Mol. Biol. 1999, 287, 627-644.

(3) Wilson, D. N.; Beckmann, R. The ribosomal tunnel as a functional environment for nascent polypeptide folding and translational stalling. Curr. Opin. Struct. Biol. 2011, 21, 274-282.

(4) Lu, J.; Deutsch, C. Folding Zones inside the Ribosomal Exit Tunnel. Nat Struct Mol Biol 2005, 12, 1123-1129. 
(5) Komar, A. A. A Pause for Thought along the Co-Translational Folding Pathway. Trends Biochem. Sci. 2009, 34, 16-24.

(6) Tu, L. W.; Deutsch, C. A Folding Zone in the Ribosomal Exit Tunnel for Kv1.3 Helix Formation. Journal of Molecular Biology 2010, 396, 1346-1360.

(7) O’Brien, E. P.; Christodoulou, J.; Vendruscolo, M.; Dobson, C. M. New Scenarios of Protein Folding Can Occur on the Ribosome. J. Am. Chem. Soc. 2011, 133, 513-526.

(8) O’Brien, E. P.; Hsu, S.-T. D.; Christodoulou, J.; Vendruscolo, M.; Dobson, C. M. Transient Tertiary Structure Formation within the Ribosome Exit Port. J. Am. Chem. Soc. 2010, 132, 16928-16937.

(9) Bhushan, S.; Gartmann, M.; Halic, M.; Armache, J.-P.; Jarasch, A.; Mielke, T.; Berninghausen, O.; Wilson, D. N.; Beckmann, R. $\alpha$-Helical Nascent Polypeptide Chains Visualized within Distinct Regions of the Ribosomal Exit Tunnel. Nat Struct Mol Biol 2010, 17, 313-317.

(10) Kaiser, C. M.; Goldman, D. H.; Chodera, J. D.; Tinoco, I.; Bustamante, C. The Ribosome Modulates Nascent Protein Folding. Science 2011, 334, 1723-1727.

(11) Bañó-Polo, M.; Baeza-Delgado, C.; Tamborero, S.; Hazel, A.; Grau, B.; Nilsson, I.; Whitley, P.; Gumbart, J. C.; von Heijne, G.; Mingarro, I. Transmembrane but Not Soluble Helices Fold inside the Ribosome Tunnel. Nature Communications 2018, 9, 5246.

(12) Liutkute, M.; Samatova, E.; Rodnina, M. V. Cotranslational Folding of Proteins on the Ribosome. Biomolecules 2020, 10, 97.

(13) Ziv, G.; Haran, G.; Thirumalai, D. Ribosome Exit Tunnel Can Entropically Stabilize Alpha-Helices. Proc. Natl. Acad. Sci. U.S.A. 2005, 102, 18956-18961. 
(14) O’Brien, E. P.; Stan, G.; Thirumalai, D.; Brooks, B. R. Factors Governing Helix Formation in Peptides Confined to Carbon Nanotubes. Nano Lett. 2008, 8, 3702-3708.

(15) Mittal, J.; Best, R. B. Thermodynamics and Kinetics of Protein Folding under Confinement. Proc. Natl. Acad. Sci. U.S.A. 2008, 105, 20233-20238.

(16) Sorin, E. J.; Pande, V. S. Nanotube Confinement Denatures Protein Helices. J. Am. Chem. Soc. 2006, 128, 6316-6317.

(17) Zhou, H.-X. Helix Formation inside a Nanotube: Possible Influence of Backbone-Water Hydrogen Bonding by the Confining Surface through Modulation of Water Activity. J. Chem. Phys. 2007, 127, 245101.

(18) Suvlu, D.; Samaratunga, S.; Thirumalai, D.; Rasaiah, J. C. Thermodynamics of HelixCoil Transitions of Polyalanine in Open Carbon Nanotubes. J. Phys. Chem. Lett. 2017, 8, 494-499.

(19) Lucent, D.; Vishal, V.; Pande, V. S. Protein Folding under Confinement: A Role for Solvent. Proc. Natl. Acad. Sci. U.S.A. 2007, 104, 10430-10434.

(20) Vaitheeswarana, S.; Thirumalai, D. Interactions between Amino Acid Side Chains in Cylindrical Hydrophobic Nanopores with Applications to Peptide Stability. Proceedings of the National Academy of Sciences of the United States of America 2008, 105, 1763617641.

(21) Vaitheeswaran, S.; Reddy, G.; Thirumalai, D. Water-Mediated Interactions between Hydrophobic and Ionic Species in Cylindrical Nanopores. J. Chem. Phys. 2009, 130, 094502.

(22) Vaitheeswaran, S.; Chen, J.; Thirumalai, D. Hydrophobic and Ionic-Interactions in Bulk and Confined Water with Implications for Collapse and Folding of Proteins. $J$ Stat Phys 2011, 145, 276-292. 
(23) Vaitheeswaran, S.; Thirumalai, D. Entropy and Enthalpy of Interaction between Amino Acid Side Chains in Nanopores. J. Chem. Phys. 2014, 141, 22 D523.

(24) Pratt, L. R.; Chandler, D. Theory of the Hydrophobic Effect. J. Chem. Phys. 1977, 67, 3683-3704.

(25) Pratt, L. R. MOLECULAR THEORY OF HYDROPHOBIC EFFECTS: "She Is Too Mean to Have Her Name Repeated.". Annu. Rev. Phys. Chem. 2002, 53, 409-436.

(26) Ball, P. Water as an Active Constituent in Cell Biology. Chem. Rev. 2008, 108, 74-108.

(27) Berne, B. J.; Weeks, J. D.; Zhou, R. Dewetting and Hydrophobic Interaction in Physical and Biological Systems. Annu. Rev. Phys. Chem. 2009, 60, 85-103.

(28) Levy, Y.; Onuchic, J. N. Water Mediation in Protein Folding and Molecular Recognition. Annu. Rev. Biophys. Biomol. Struct. 2006, 35, 389-415.

(29) Bellissent-Funel, M.-C.; Hassanali, A.; Havenith, M.; Henchman, R.; Pohl, P.; Sterpone, F.; van der Spoel, D.; Xu, Y.; Garcia, A. E. Water Determines the Structure and Dynamics of Proteins. Chem. Rev. 2016, 116, 7673-7697.

(30) Reddy, G.; Straub, J. E.; Thirumalai, D. Dry Amyloid Fibril Assembly in a Yeast Prion Peptide Is Mediated by Long-Lived Structures Containing Water Wires. Proc Natl Acad Sci U S A 2010, 10\%, 21459-21464.

(31) Thirumalai, D.; Reddy, G.; Straub, J. E. Role of Water in Protein Aggregation and Amyloid Polymorphism. Acc. Chem. Res. 2012, 45, 83-92.

(32) Pohorille, A.; Pratt, L. R. Is Water the Universal Solvent for Life? Orig Life Evol Biosph 2012, 42, 405-409.

(33) Hummer, G. Molecular Binding: Under Water's Influence. Nature Chemistry 2010, 906-907. 
(34) Setny, P.; Baron, R.; McCammon, J. A. How Can Hydrophobic Association Be Enthalpy Driven? J. Chem. Theory Comput. 2010, 6, 2866-2871.

(35) Durell, S. R.; Ben-Naim, A. Hydrophobic-Hydrophilic Forces in Protein Folding. Biopolymers 2017, 10\%, e23020.

(36) Kopel, Y.; Giovambattista, N. Comparative Study of Water-Mediated Interactions between Hydrophilic and Hydrophobic Nanoscale Surfaces. J. Phys. Chem. B 2019, 123, $10814-10824$.

(37) Kanduč, M.; Schlaich, A.; Schneck, E.; Netz, R. R. Water-Mediated Interactions between Hydrophilic and Hydrophobic Surfaces. Langmuir 2016, 32, 8767-8782.

(38) Shin, S.; Willard, A. P. Characterizing Hydration Properties Based on the Orientational Structure of Interfacial Water Molecules. J. Chem. Theory Comput. 2018, 14, 461-465.

(39) Shin, S.; Willard, A. P. Water's Interfacial Hydrogen Bonding Structure Reveals the Effective Strength of Surface-Water Interactions. J. Phys. Chem. B 2018, 122, 67816789.

(40) Hummer, G.; Rasaiah, J. C.; Noworyta, J. P. Water Conduction through the Hydrophobic Channel of a Carbon Nanotube. Nature 2001, 414, 188-190.

(41) Waghe, A.; Rasaiah, J. C.; Hummer, G. Filling and Emptying Kinetics of Carbon Nanotubes in Water. J. Chem. Phys. 2002, 117, 10789.

(42) Vaitheeswaran, S.; Yin, H.; Rasaiah, J. C.; Hummer, G. Water Clusters in Nonpolar Cavities. Proc. Natl. Acad. Sci. U.S.A. 2004, 101, 17002-17005.

(43) Rasaiah, J. C.; Garde, S.; Hummer, G. Water in Nonpolar Confinement: From Nanotubes to Proteins and Beyond *. Annu. Rev. Phys. Chem. 2008, 59, 713-740.

(44) Sugita, Y.; Okamoto, Y. Replica-Exchange Molecular Dynamics Method for Protein Folding. Chemical Physics Letters 1999, 314, 141-151. 
(45) Abraham, M. J.; Murtola, T.; Schulz, R.; Páll, S.; Smith, J. C.; Hess, B.; Lindahl, E. GROMACS: High Performance Molecular Simulations through Multi-Level Parallelism from Laptops to Supercomputers. SoftwareX 2015, 1-2, 19-25.

(46) Best, R. B.; Mittal, J.; Feig, M.; Mackerell, A. D. Inclusion of Many-Body Effects in the Additive CHARMM Protein CMAP Potential Results in Enhanced Cooperativity of $\alpha$-Helix and $\beta$-Hairpin Formation. Biophys. J. 2012, 103, 1045-1051.

(47) Huang, J.; MacKerell, J., Alexander D CHARMM36 All-Atom Additive Protein Force Field: Validation Based on Comparison to NMR Data. J. Comput. Chem. 2013, 34 , $2135-2145$.

(48) Patriksson, A.; van der Spoel, D. A Temperature Predictor for Parallel Tempering Simulations. Phys. Chem. Chem. Phys. 2008, 10, 2073.

(49) Hess, B. Determining the Shear Viscosity of Model Liquids from Molecular Dynamics Simulations. J. Chem. Phys. 2002, 116, 209.

(50) Rueda, M.; Ferrer-Costa, C.; Meyer, T.; Pérez, A.; Camps, J.; Hospital, A.; Gelpí, J. L.; Orozco, M. A Consensus View of Protein Dynamics. PNAS 2007, 104, 796-801.

(51) Meyer, T.; Gabelica, V.; Grubmüller, H.; Orozco, M. Proteins in the Gas Phase. Wiley Interdisciplinary Reviews: Computational Molecular Science 2013, 3, 408-425.

(52) Ben-Amotz, D.; Raineri, F. O.; Stell, G. Solvation Thermodynamics: Theory and Applications. J. Phys. Chem. B 2005, 109, 6866-6878.

(53) Ben-Amotz, D.; Underwood, R. Unraveling Water's Entropic Mysteries: A Unified View of Nonpolar, Polar, and Ionic Hydration. Acc. Chem. Res. 2008, 41, 957-967.

(54) Ben-Amotz, D. Water-Mediated Hydrophobic Interactions. Annu. Rev. Phys. Chem. 2016, 67, 617-638. 
(55) Ben-Amotz, D. Interfacial Solvation Thermodynamics. J. Phys.: Condens. Matter 2016, 28, 414013-11.

(56) Lifson, S.; Roig, A. On the Theory of Helix - Coil Transition in Polypeptides. J. Chem. Phys. 1961, 34, 1963-1974.

(57) Willard, A. P.; Chandler, D. The Role of Solvent Fluctuations in Hydrophobic Assembly. J. Phys. Chem. B 2008, 112, 6187-6192.

(58) Willard, A. P.; Chandler, D. The Molecular Structure of the Interface between Water and a Hydrophobic Substrate Is Liquid-Vapor Like. J. Chem. Phys. 2014, 141, 18 C519.

(59) Papoian, G. A.; Ulander, J.; Eastwood, M. P.; Luthey-Schulten, Z.; Wolynes, P. G. Water in Protein Structure Prediction. PNAS 2004, 101, 3352-3357.

(60) Davtyan, A.; Schafer, N. P.; Zheng, W.; Clementi, C.; Wolynes, P. G.; Papoian, G. A. AWSEM-MD: Protein Structure Prediction Using Coarse-Grained Physical Potentials and Bioinformatically Based Local Structure Biasing. J. Phys. Chem. B 2012, 116, 8494-8503. 
bioRxiv preprint doi: https://doi.org/10.1101/2020.11.11.378026; this version posted November 11,2020 . The copyright holder for this preprint (which was not certified by peer review) is the author/funder, who has granted bioRxiv a license to display the preprint in perpetuity. It is made available under aCC-BY-NC 4.0 International license.

\section{Graphical TOC Entry}

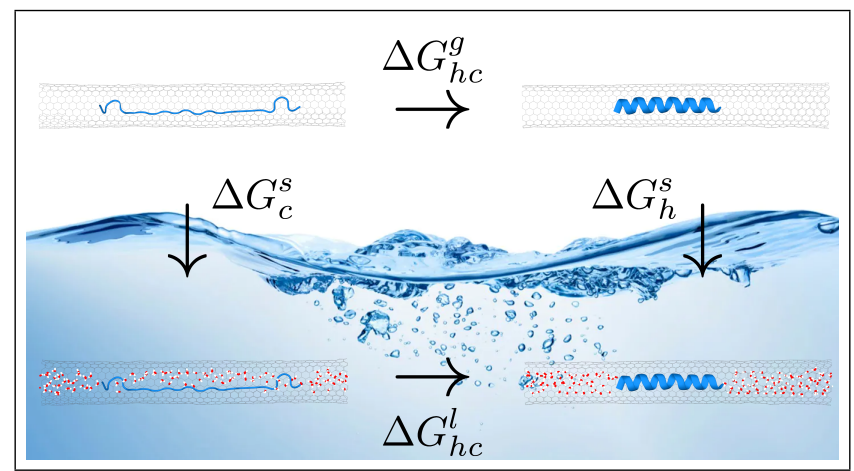

